\title{
THE PRACTICE OF ORDER-CULTIVATION OF TALENTS IN COLLEGES AND UNIVERSITIES OF HUBEI PROVINCE
}

\author{
Ke Xianzhu ${ }^{1 \#}$ Tian FuMing $1^{\#}$ Li Fei $^{1}$ \\ Department of Medical Affair Renmin Hospital, \\ Hubei University of medicine Shiyan 442000
}

\begin{abstract}
Order-orientated cultivation of talents is an effective, distinctive model for universities, which can help cultivating students' employment ability and solve the employment problem. As for some of the problems exposed in the practice of order-orientated cultivation of talents in some medical college in Hubei province such as the application quantity being inconsistent with the actual needs, the weak implementation of training plans, unclear career planning objectives and the unscientific assessment by leaders.
\end{abstract}

This paper discusses the countermeasures as follows: improving the ability to predict talent demand, establishing dynamic screening system to avoid the risk of the process of talent cultivation, using the methods of two-way choice and wide into severe out, giving priority to the using of outstanding local talents, increasing the humanistic quality training, using a variety of forms to complete training plans, offering help to make career planning, promoting the realization of vision, establishing a scientific evaluation system, strengthening the construction of training base and so on, so that to alleviate the contradiction between supply and demand of human resources.

Keywords - Order-cultivation of talents; Problem countermeasure

\section{INTRODUCTION}

The so-called order-orientated talents training mode refers to the demand side (usually enterprises and institutions) and offer (mainly the schools) for consultations, proposed by the provider according to the demand of talents needed in quantity, level of knowledge and vocational skills requirements, Demand within the specified time which shall submit a corresponding quantity and quality of human resources contract [1]. Order- orientated training pattern in many colleges and universities are considered to be an effective, featured training mode of students ' employability, it has been recognized by the ministries of education, educational experts, as well as vocational education workers to solve the employment problem, and is now considered to be models of schooling patterns for higher education with the promotion phase [2]. In Hubei Province, a medical college

\author{
Wang Qian ${ }^{2 *}$ \\ Center of Health Administration and Development Studies, Hubei \\ University of Medicine 442000
}

and affiliated hospitals in the past two years try to order training. This paper presents case of the two-year training practice through analyzing the training present situation, existing problems and propose appropriate solutions.

\section{ORDERS TRAINING OF MEDICAL TALENTS}

\section{1 order training objects}

From 2012 year, first half of April per year, from the human resources Department of the hospital, the internship grade the top 120 students in specialized filters (mainly clinical, nursing) were chosen by the Medical Department. (Their English scores are the above priority, according to a written application on a personal, then combination of theory and basic skills assessment, sorted by scores identified 60 students training were chosen as a key objects. They are trained by internship training agreements with hospital human resources department where interns selected order training provided by the hospital every month 300 internship subsidies, give priority to key specialist internship and free hospital for all kinds of training.

\section{2 orders, personnel training and assessment methods 1.3}

Selected focus groups are taken into specialist or directional positions (such as Pediatrics, emergency medicine, cardiology, ICU specialist), and rotation culture, 2-4 week rotation, they learn medical skills in critically ill patients, and each specialist is trained to develop appropriate training plans, specialty medical technology program, medical most common illnesses, the number of serious cases. Trained students must complete internships and 8 rotation directional posts after a key specialist of 3 months internship, it finalizes the position. During the internship, training departments and the Hospital organized various training, supervised the thesis research. After the end of each rotation time, theory skill assessment, comprehensive quality assessment, dynamic management of the Department of medical services, care, survival of the 
fittest will be proceeded, and incompetent or unfit to continue as training objects will be discarded in a timely manner. After comprehensive evaluation before the end of the internship, the students with 8 specialist examination results with average score $70 \%+$, directed Department evaluation $20 \%+, 10 \%$ evaluation of sorts will be as taken as hiring candidates, and sign the formal contract of employment.

\section{ORDER PROBLEMS IN THE CULTIVATION OF TALENTS}

\section{1 employment numbers is inconsistent with actual demand}

Annually on the basis of actual needs 60 people signed agreement, but the actual employment rate is less than $60 \%$. Main reasons: first, the internees with signing training agreement during the internship is discarded due to bad scores; second, they select employment in home town or in economically developed regions; the third, they was admitted to graduate school to give up job opportunities. Hospital human resource supply was complained by hospitals employing departments, thus thinking about that previous graduate's recruitment of talent is much more direct, more convenient.

\subsection{Implementation training plan is insufficient, still need to improve students ' comprehensive quality and ability}

Internship plans of Clinical and nursing professional overall are bias to clinical practice capacity improvement, although set of full is detailed, but hard to implement; the section assessment content and way are not same, assessment lacks uniform; from part students obviously out medical professional of talent training also is not suit to social needs of changes; Medical Humanities aspects of training and industry requirements is not match, lacking Humanities literacy capacity upgrade, limited capacity of communication and solution complex problem o, unable to keep up with medical comprehensive ability, and sense of service can't keep up with changes in health service; this medical talent training detached with social needs is difficult to solve in a short time. Apparently, order training students currently receive occupational skill needs, while it is not fully up to the development of students ' comprehensive ability.

\section{3 career planning objectives are not clear}

Bachelor of clinical $4+1$, Bachelor of nursing $3+1$ course mode bring in the detach of theoretical knowledge with clinical skills, make order-oriented talent to choose to give up clinical practice, and continue to apply postgraduate position, and clinical practice quality cannot be guaranteed. And actual orders-internship teacher s' abilities are not the same, some with practice can not teach well. Order training is required to address employment, improve the ability of comprehension and practice, but the accumulation of clinical practice experience doesn't happen overnight, this is the short-term training cannot solve the problem.

\section{4 leadership assessment is not scientific}

Hospital in dynamic assessment sometimes is affected by leaders' employing decision status, for example, $10 \%$ of evaluation score can be controlled by hospital leaders; due to that hospital leaders and internship students in actual work does not contact, and not understand, results only is given by impression scoring, this will cause evaluation score without scientific, even some parents to children supporting relationship.

\section{STRATEGY}

3.1 improve the talent demand prediction, the establishment of "dynamic filters" cover [3] Talent cultivation process risk

Throughout the process of personnel training, monitoring students ' learning processes and attitudes is used to adjust students according to the actual situation at any time. This can prevent the insufficient number of orders, encourage students who have no contracts to participate in the competition. In the other hand, the students, with the migration over time, and fluctuations in employment intentions, does not rule out selecting grind or seek employment in other hospitals, and "dynamic filter system" could provide an opportunity for these students, or for hospitals to receive qualified satisfaction with the number of training strategy adjustments in a timely manner. In the course of training, human resources, medical services, nursing, teaching, clinical Department joint implementation training plan, transform order-talent theory to clinical practice, develop potential to meet hospital needs, cultural needs. For accurate human resources needs planning, the hospital's decision provides accurate and timely information for decision-making.

\section{2 setting up scientific evaluation system, and strengthen the construction of practical training base}

We have suggestions such as cancelling leadership impression scores, creating order-internship archives; developing detailed training plans with strict three-base training. Students can check the quantitative results to stimulate self-esteem, pride, and it will form orderly competition.

To sum up, human resource management is one of the core management of hospital orders; personnel training is adapting to the affiliated teaching hospitals characteristic way, in response to rapid development of hospital personnel needs; 
Order-oriented talent have strong plasticity, it is good try for medical institutions, hospitals, and medical students; Hospitals and medical institutions need to work together in the future, and constantly sum up and improve personnel training in the practice.

\section{REFERENCES}

[1] Luo Hong. Vocational "order type" limitations of talent training mode and consummation [j]. Journal of yunyang teachers college 2007,1 (4): 118

[2] Zhou Peiming. Analysis of factors influencing higher vocational students ' ability to [j]. Vocational education forum 2009, 1(1):55

[3] Guan you bridge. "Order form" predicament and countermeasure of talents training mode of [j]. Education and professional 2009, 1(12)4:15

[4] Feng Ruolei. "Two in one" training target practice and consideration of construction [j]. Residency training

[5] Bai Xiaoling, louting, dachun, and so on. Based on Confucian humanism caring in nursing study on the evaluation index system [j]. Journal of nursing 2012, 27(11):58-61

[6] Li Kaijun, Cui Rongjun and $\mathrm{Hu}$ Jiangping. Problems of medical humanistic education under the new situation and countermeasure of China [j]. Medicine and society 2013, 26(3):89-90

[7] Jie Fuquan. The "order-training" reflections [j]. China's vocational and technical education in 2006, 1(3):7

About the author: These authors with Sign\# contributed equally to this work. These author with Sign* is Corresponding author.

This paper is supported by key research bases of Humanities in colleges and universities of Hubei Province opened on funded projects 